\title{
Preauricular Incision Outlining During a Face-Lift: A Step-by-Step Description
}

\author{
Kahraman Berkhan Yilmaz
}

Received: 23 November 2012/ Accepted: 30 December 2012/Published online: 22 March 2013

(C) Springer Science+Business Media New York and International Society of Aesthetic Plastic Surgery 2013

Level of Evidence $V$ This journal requires that authors assign a level of evidence to each article. For a full description of these Evidence-Based Medicine ratings, please refer to the Table of Contents or the online Instructions to Authors www.springer.com/00266.

I read the article, Preauricular incision outlining during a face-lift: a step-by-step description, by Albertal et al. [1] with great interest. Postoperative distortion in the preauricular region remains a significant problem in face-lift procedures. I applaud the authors for providing a substantial review of the concepts and technique required for the design of an appropriate preauricular incision during primary rhytidoplasty. In addition they have presented their operative technique for helix, tragus, and earlobe incisions in an attempt to avoid distortions in this region.

This article is a great addition to the existing literature on the subject. However, for the sake of completeness, we comment on the term "submucosal aponeurotic system (SMAS)" used by the authors in the Materials and Methods section. This seems to be a typographical error. We believe that the authors meant the superficial musculoaponeurotic system and hence the acronym SMAS. In addition, this little correction may eliminate potential confusion among young plastic surgeons.

\section{Reference}

1. Albertal JM et al (2012) Preauricular incision outlining during a face-lift: a step-by-step description. Aesthetic Plast Surg 36: $857-861$
K. B. Yilmaz ( $\square)$

Plastic Reconstructive and Aesthetic Surgery, Acibadem

University, Acibadem Hospital, Tekin Sok No. 8,

Acibadem-Kadikoy, 34718 Istanbul, Turkey

e-mail: berkhany@hotmail.com 